MCEWAN Colin and Leonardo LÓPEZ LUJÁN (eds), Moctezuma Aztec Ruler

\title{
Maëlle Sergheraert
}

\section{OpenEdition}

\section{Journals}

Édition électronique

URL : https://journals.openedition.org/jsa/11409

DOI : 10.4000/jsa. 11409

ISSN : 1957-7842

\section{Éditeur}

Société des américanistes

\section{Édition imprimée}

Date de publication : 5 juin 2010

Pagination : 297-300

ISSN : 0037-9174

\section{Référence électronique}

Maëlle Sergheraert, « mcewan Colin and Leonardo López luján (eds), Moctezuma Aztec Ruler », Journal de la Société des américanistes [En ligne], 96-1 | 2010, mis en ligne le 11 juillet 2010, consulté le 03 septembre 2022. URL : http://journals.openedition.org/jsa/11409; DOI : https://doi.org/10.4000/jsa. 11409

Ce document a été généré automatiquement le 3 septembre 2022

Tous droits réservés 


\title{
MCEWAN Colin and Leonardo LóPEZ LUJÁN (eds), Moctezuma Aztec Ruler
}

\author{
Maëlle Sergheraert
}

\section{RÉFÉRENCE}

MCEWAN Colin and Leonardo LóPEZ LUJÁN (eds), Moctezuma Aztec Ruler, The British Museum Press, Londres, 2009, 320 p., bibl., gloss., notes, index, ill. coul.

1 De septembre 2009 à janvier 2010, le British Museum consacrait une exposition à Moctezuma Xocoyotzin (dernier souverain élu de l'empire aztèque), bouclant ainsi un cycle de quatre expositions consacrées aux grands hommes de pouvoir de l'histoire universelle ${ }^{1}$. On pouvait ainsi admirer sous la rotonde du musée un ensemble de 131 objets préhispaniques et coloniaux permettant d'examiner «la vie, le règne et la mort controversée de ce souverain pendant la conquête espagnole ».

2 En écho à l'événement, Colin McEwan et Leonardo López Luján ont publié un catalogue illustré en quadrichromie de très belle facture, dépassant la simple fonction de répertoire des œuvres exposées, pour devenir un intéressant complément scientifique de l'exposition. L'ouvrage, intitulé Moctezuma Aztec Ruler, fait en effet appel à vingt-et-un chercheurs spécialistes du monde aztèque pour proposer, à travers une série de huit chapitres thématiques (cinq consacrés à la période préhispanique, trois à la période coloniale), une véritable étude de divers aspects du règne de Moctezuma Xocoyotzin. Une introduction, un épilogue prolongé par un glossaire, un glossaire des glyphes, les notes des différents chapitres, une bibliographie et un index complètent l'ensemble.

Sur le plan formel, les auteurs ont pris le parti d'illustrer leurs propos, en cours de chapitre avec leur propre matériel documentaire et à la fin de chacun d'eux avec une série de planches présentant les pièces de l'exposition en lien avec le thème étudié (au total 98 figures et les photographies des 131 pièces exposées). Les commentaires scientifiques associés à chacune des œuvres rehaussent l'intérêt de la consultation de ces feuillets. Ils se composent, dans l'ordre, d'un volet informatif (date approximative de 
production, matériau, dimensions, lieu de conservation), d'un encart très utile renvoyant à une bibliographie sélective et d'une description rédigée par l'un des vingt-et-un contributeurs, à même d'éclairer, selon son domaine de spécialité, tel ou tel aspect de l'œuvre (style, approche symbolique et fonctionnelle, technique de production...). Le seul petit regret au sujet de ces commentaires concerne la perte des excellents schémas explicatifs présents dans l'exposition qui éclairaient l'iconographie de certaines œuvres. Ajoutons que, pour la bonne compréhension du lecteur n'ayant pas eu la chance de visiter l'exposition, il eût été utile de stipuler plus clairement, en tête des feuillets de fin de chapitre, que les objets photographiés étaient ceux présentés au British Museum.

L'introduction du catalogue, rédigée par Colin McEwan et Leonardo López Luján, nous entraîne dans le monde des Aztèques - ou plus exactement des Mexica - et situe rapidement le contexte du règne de Moctezuma Xocoyotzin. Elle présente également les sources informatives sur le sujet et souligne l'intérêt croissant des données archéologiques qui complètent peu à peu le panel des données écrites. Elle expose enfin la visée de la publication: tenter de reconsidérer objectivement l'histoire de Moctezuma, si souvent tiraillée entre points de vue européen et mexicain.

Le premier chapitre, écrit par Felipe Solís Olguín, propose un condensé de l'histoire des Mexica : il est d'abord question d'Aztlan, le lieu mythique de leur origine, puis de leur pérégrination, de leur installation dans la vallée de Mexico et de la généalogie des souverains ayant précédé Moctezuma Xocoyotzin. L'intérêt de cette étude réside dans l'effort d'actualisation réalisé par l'auteur et dans sa prise en compte, pour relater les événements, tant des découvertes archéologiques que des documents écrits. Outre le fait qu'il dresse une rétrospective assez vivante de l'histoire mexica, ce chapitre nous éclaire aussi sur le système de succession des souverains et les tactiques d'alliances matrimoniales destinées à renforcer le pouvoir de l'empire. Les treize premières œuvres exposées au British Museum (parmi lesquelles plusieurs se rapportent à la fondation de Tenochtitlan) constituent les feuillets commentés de la fin du chapitre.

Les quatre chapitres suivants sont consacrés au règne de Moctezuma Xocoyotzin proprement dit. Dans le chapitre II, Eduardo Matos Moctezuma s'intéresse au début du règne en évoquant les qualités ayant conduit au choix de cet homme, puis les célébrations ayant précédé son avènement. Notons d'ailleurs que la pierre supposée commémorer cet événement apparaît dans les feuillets de fin de chapitre. Eduardo Matos Moctezuma s'attache ensuite à décrire le palais de Moctezuma et les différentes activités qui s'y déroulaient. Si son récit, fondé essentiellement sur le témoignage de documents écrits, n'a rien de vraiment novateur, les photographies de certains éléments de décor du palais (sculptures, frises architecturales...) permettent de s'en faire une idée plus précise.

7 Dans le chapitre III, Guilhem Olivier et Leonardo López Luján abordent la question des modes de représentations de Moctezuma. Contrairement aux Européens, les Mexica ne pratiquaient pas l'art du portrait et l'on reconnaît la figure de Moctezuma à travers le glyphe de son nom que les auteurs décrivent fort bien, mais dont on aurait aimé voir un dessin. Puis, ils passent en revue les différents attributs symboliques des souverains mexica (religieux, politiques et guerriers). Une fois de plus, les photographies des œuvres exposées jouent un rôle essentiel pour mieux se les représenter.

Dans le chapitre IV, Richard F. Townsend rappelle que la religion des Mexica est fortement influencée par leur environnement naturel. Après une brève description de leur vision cosmologique et de l'enceinte sacrée du Templo Mayor, il explique quel était le rôle joué par le souverain, notamment lors de deux fêtes importantes : les rites annuels 
au mont Tlaloc et la cérémonie du Feu nouveau. Cette dernière, célébrée seulement tous les 52 ans, fut l'un des grands événements du règne de Moctezuma Xocoyotzin en 1507 ; le monolithe sculpté qui l'a commémoré constituait d'ailleurs la pièce centrale de l'exposition. Au fil des «feuillets exposition» de fin de chapitre, on découvre couteau sacrificiel, boîtes en pierre sculptées, encensoir et sculptures de divinités dont un certain nombre en mosaïque de turquoise, à l'instar du masque représenté en couverture du catalogue.

Le chapitre $\mathrm{v}$ est consacré au gouvernement militaire et économique de Moctezuma.

Frances F. Berdan y décrypte la stratégie suivie par le souverain, à la fois pour consolider un empire qui s'est considérablement étendu lors des règnes précédents, mais aussi pour en tirer le maximum de profit, notamment en denrées de luxe (or, jade, turquoise, cacao, plumes...). Les «feuillets exposition» de fin de chapitre révèlent des armes utilisées sur le champ de bataille, des sculptures représentant des guerriers et des denrées demandées en tribut.

Ces cinq premiers chapitres, à partir de cinq éclairages, témoignent des 19 années de règne du dernier souverain élu des Mexica. Si les éléments y figurant ne constituent pas des nouveautés, ils ont le mérite de synthétiser les dernières connaissances sur le sujet, d'offrir au grand public une très bonne qualité de vulgarisation et aux chercheurs, à travers le commentaire des objets, des informations beaucoup plus pointues. L'ouvrage marque alors un tournant, sensible également dans l'exposition, avec les trois derniers chapitres où sont examinées la période d'arrivée des Espagnols et les répercussions de la conquête des siècles plus tard et jusqu'à aujourd'hui, dans le regard porté sur Moctezuma.

Dans le chapitre VI, John H. Elliott reprend le fil des événements tels qu'ils se sont déroulés, ou, plutôt, tels qu'ils ont ensuite été rapportés : présages, débarquement des Espagnols sur la Côte du Veracruz, entrée dans Tenochtitlan, emprisonnement et mort de Moctezuma, fuite des Espagnols et siège de Tenochtitlan, jusqu'à la victoire espagnole le 13 août 1521. L'auteur veille à conserver ses distances vis-à-vis des sources disponibles, car celles-ci sont nécessairement subjectives et reflètent soit le point de vue espagnol, soit le point de vue mexica. Ainsi, il ne tranche délibérément par sur les circonstances de la mort de Moctezuma du fait de la diversité des témoignages. Concernant l'iconographie de fin de chapitre tirée de l'exposition, il s'agit de nombreuses pièces de l'époque coloniale, dont une série de tableaux illustrant les événements, le tout s'ajoute aux pièces préhispaniques qui constituaient jusque-là l'essentiel des œuvres exposées.

Dans le chapitre VII, David A. Brading explore ce qu'il appelle la renaissance de l'ancienne Mexico. Il énumère les différents types d'ouvrages rédigés au fil des $\mathrm{xVI}^{\mathrm{e}}$, $\mathrm{XVII}^{\mathrm{e}}$ et $\mathrm{XVIII}^{\mathrm{e}}$ siècles à propos de la culture mexica, dont il envisage l'aspect identitaire au regard de la future nation mexicaine.

Enfin, le dernier chapitre, dû à Salvador Rueda Smithers et intitulé "Rethinking Moctezuma», prend le parti singulier de s'interroger sur la perception que le monde moderne a du souverain. Longtemps tenu pour responsable de la défaite mexica face aux conquistadors et cantonné au rôle de traître à sa patrie, Moctezuma est peu à peu perçu de façon plus nuancée quand on prend en compte les complexités qu'impliquait la situation historique.

L'épilogue de l'ouvrage se présente moins comme une conclusion que comme une ouverture à l'actualité archéologique, puisqu'il est question des récentes découvertes 
effectuées au Templo Mayor de Mexico, qui pourraient nous renseigner sur la manière dont les souverains mexica étaient inhumés. Ce qui reste encore assez méconnu et complèterait pertinemment nos connaissances concernant les rites et traditions entourant la mort des grands souverains mexica.

$\mathrm{Au}$ terme de notre lecture, il apparaît que ce catalogue documenté de l'exposition Moctezuma Aztec Ruler constitue indéniablement une somme d'informations sérieuses pour un public curieux d'étoffer son savoir. La communauté scientifique avait, pour sa part, déjà découvert le personnage de Moctezuma au travers de l'excellente publication de Michel Graulich en 1994. C'est donc moins dans les articles de synthèse du catalogue qui ne vont pas au-delà des connaissances synthétisées et discutées par Graulich - que dans le fonds iconographique et les fiches documentaires qui l'accompagnent, que les chercheurs trouveront un très précieux apport. Cette dimension visuelle, qui manquait cruellement jusqu'alors, contribue enfin à nous rendre concret le règne de Moctezuma.

\title{
BIBLIOGRAPHIE
}

\author{
GRAULICH Michel
}

1994 Montezuma ou l'apogée et la chute de l'empire aztèque, Fayard, Paris.

\section{NOTES}

1. Les trois premières, "The first emperor ", "Hadrian" et "Shah'Abbas ", étaient respectivement consacrées au premier empereur de Chine, à l'empereur romain Hadrien et au souverain iranien le plus remarquable de la dynastie safavide.

\section{AUTEURS}

\section{MAËLLE SERGHERAERT}

Docteur en préhistoire, ethnologie, anthropologie, Université Paris 1, Panthéon-Sorbonne 